\title{
Progressive multifocal leucoencephalopathy
}

\author{
S. B. CHANDOR, L. S. FORNO, AND N. A. WIVEL \\ From the Departments of Medicine (Neurology) and Pathology, Veterans Administration Hospital and Stanford \\ University, Palo Alto, California
}

In 1958, a disease process termed progressive multifocal leucoencephalopathy was described by Åström, Mancall, and Richardson. They presented three cases and reviewed the literature, finding five more which fulfilled their criteria. Thereafter, sporadic cases were reported, and in 1961 Richardson reviewed these cases and added more from his own experience, bringing the total of documented cases to 22 . He discussed the various clinical and pathological features of the disease, and described one new microscopic finding common to three cases, namely, alteration of the nuclei in the deeper portion of the cerebellar granular layer. Since then several new case reports have appeared, but the disease remains a rare one with a poorly understood aetiology (Hecker and Reid, 1962). Each new case deserves to be recorded. We wish to present two additional cases. One demonstrated no symptoms during life which could be attributed with certainty to this disease. It showed scattered, small, presumably early lesions at post-mortem examination. The other case, in contrast, demonstrated the fully developed clinical and pathological picture.

\section{CASE REPORTS}

CASE 1 A.M., a Caucasian woman, was transferred from a state mental institution to the Palo Alto Veterans Administration Hospital in April 1961, at the age of 82 . She had been active as a registered nurse most of her adult life and had essentially no medical history until she was involved in an automobile accident in August 1959. She was briefly admitted to a hospital for observation and treatment of fractured ribs. There was no evidence of head injury. After discharge she became neglectful of her personal appearance and household, and she began to drink. She also had paranoid ideas about her neighbours and was admitted to a mental hospital later that year. At that time she was fully orientated but aware that she could not care for herself. She often became confused, thinking that her belongings had been stolen, her house raided by intruders, and imagining that something horrible was about to happen to her. In December 1960, she had a bout of pneumonia; following this she was weak, confused, unsteady on her feet, and her memory appeared worse.
At the time of transfer to the Veterans Administration Hospital four months later, she was a pleasant, neat, cooperative elderly woman who was disorientated in regard to place, time, and person. She could recall very little of her previous life. During the examination she appeared to have spells of dizziness during which she would push people away and lean against the wall. Physical examination revealed slight cardiac enlargement. Her blood pressure was normal, but on other occasions it was sometimes elevated up to $210 / 110 \mathrm{~mm}$. Hg. Her pulse and temperature were normal. There was a midline lower abdominal scar, the reason for which was never established. She appeared somewhat emaciated and weak, but she could move all her extremities freely. The deep $c$ tendon reflexes were present and equal. A 'senile' tremof i was noted. Her gait was unsteady with occasional goose step to the left and a tendency to propulsion. The haemg tocrit was $41 \%$, haemoglobin $12.5 \mathrm{~g} . / 100 \mathrm{ml}$., white bloof cell count $6,800 /$ c.mm. $(66 \%$ polymorphonuclear leucs $c$ cytes, $1 \%$ bands, and $33 \%$ lymphocytes). There was trace proteinuria. The blood serology was negative.

During the following two years she remained in the $\overrightarrow{0}$ hospital and was treated with various drug regimense of including meprobamate, Thioridazine, phenobarbital, and prochlorperazine. Her mental status fluctuated between periods when she was pleasant and cooperative and intervals when she was markedly hostile, negativistic, and combative. Her memory continued to deteriorate. She was quite unsteady on her feet and had frequent falls. This unsteadiness was, at least at times, associated with episodes of cardiac arrythmias, for which she was evaluated on the medical service. Electrocardiograms (E.C.G.) revealed atrial bigemini with aberrant conduction shortly after admission and later atrial fibrillation or flutter. She was digitalized with temporary improvement. During the most severe of these episodes, in August 1961, she was confused, acted inappropriately, and seemed to have slurred speech. The right eyelid and angle of the mouth drooped; there was rigidity of both arms, but she was able to move them freely. No abnormal reflexes were elicited, and the pupils reacted to light and accommodation and were normal in size and equal. The spinal fluid examined at this time was under normal pressure, clear, colourless, and contained 1 lymphocyte/c.mm. and a few red blood cells. Spinal fluid protein was $46 \mathrm{~g} . / 100 \mathrm{ml}$. All signs cleared during one week of bed rest.

In a fall in February 1963, the patient sustained a fracture of the left femur with minimal displacement. This occurred at a time when she had been getting pro- 
gressively weaker and less active. She had been eating less and less, often refusing food completely. She was treated with bed rest for the fracture and was transferred to the medical service on 20 March when she developed cough and fever. Physical examination at this time revealed a very hostile, pale, thin, elderly woman who appeared acutely and chronically ill. Her blood pressure was $100 / 50$ $\mathrm{mm}$. $\mathrm{Hg}$, pulse 180 and irregular, and temperature $102^{\circ} \mathrm{F}$. Firm, enlarged lymph nodes were found in the neck, axilla, and inguinal regions. The liver was palpated $4 \mathrm{~cm}$. below the right costal margin. Small perirectal abcesses were present. She was able to move all extremities, and reflexes were equal and active.

Because of the pallor and anorexia, her haematological status had been followed for some time, and the white blood count had been gradually increasing, with a progressively abnormal differential count. On 4 March, when the first abnormality was noted, the white count was $3,700 /$ c.mm., with $52 \%$ polymorphonuclear leucocytes, $6 \%$ blast forms, $1 \%$ promyelocytes, $33 \%$ lymphocytes, and $8 \%$ monocytes. On 19 March the white count was $12,600 /$ c.mm., with $21 \%$ mature polymorphonuclear leucocytes, $33 \%$ blast forms, $5 \%$ myelocytes, $11 \%$ metamyelocytes, $7 \%$ promyelocytes, $8 \%$ bands, $14 \%$ lymphocytes, and $1 \%$ monocytes. The haematocrit was $33 \%$ and haemoglobin $10.9 \mathrm{~g} . / 100 \mathrm{ml}$. The spinal fluid was within normal limits with 1 lymphocyte, 7 red cells, and protein of $31 \mathrm{~g} . / 100 \mathrm{ml}$. The E.C.G. demonstrated atrial flutter with 2:1 A-V block. Analysis of the urine showed 2 plus proteinuria, bacteriuria, and 20-30 white blood cells per high-power field with occasional clumps. The blood urea nitrogen was $36 \mathrm{mg} . / 100 \mathrm{ml}$., and fasting blood sugar was $112 \mathrm{mg}$./100 ml. Electrolytes and serum transaminase levels were within the normal range. One blood culture out of four grew coagulase-negative staphylococcus, while culture of the rectal abcess showed coagulase-positive staphylococcus, E. coli, and Pseudomonas. Bone marrow smears and section revealed a hypercellular marrow with myeloid hyperplasia and prominence of unclassified blasts. Red cell precursors, megakaryocytes, and iron were not appreciably decreased. The patient was treated with penicillin and intravenous fluids, with no appreciable change in her clinical state.

Over the next week the white cell count dropped to $7,800-8,700 / \mathrm{c} . \mathrm{mm}$., but the percentage of immature myeloid cells and blast forms slowly increased. During this time the haematocrit dropped to $26 \%$, while platelet counts remained about $250,000 / \mathrm{c} . \mathrm{mm}$. During the last week of life she was placed on norethandrolone $10 \mathrm{mg}$., b.i.d. Three units of blood brought the haematocrit up to $46 \%$. Until the last few days she remained conscious, but was withdrawn and often refused to eat. During the last few days she became oliguric and blood urea nitrogen rose to $11 \mathrm{mg}$. $/ 100 \mathrm{ml}$. and potassium to $6.2 \mathrm{mEq}$./1. Terminally, there was an episode of rectal bleeding, and the white blood count rose to $44,800 / \mathrm{c}$.mm, with $18 \%$ polymorphonuclear leucocytes, $61 \%$ blast-forms, $7 \%$ myelocytes, $1 \%$ promyelocytes, $5 \%$ bands, $7 \%$ lymphocytes, and $1 \%$ monocytes. She expired quietly on 11 April 1963, exactly two years after admission to the Veterans Administration Hospital and three and one-half years after the onset of mental symptoms.
Necropsy The post-mortem examination (PAVA 63-A-76) was performed 20 hours after death. The body was that of an emaciated elderly female. The pleural spaces contained $200 \mathrm{ml}$. of cloudy yellow fluid, and the lungs were congested and focally consolidated. The heart weighed $350 \mathrm{~g}$., and the coronary vessels contained mild to moderate atherosclerosis with no evidence of recent occlusion. There was moderate atherosclerosis of the aorta, most pronounced in the aneurysmally dilated distal portion. However, all major branches had clear orifices and showed only mild atherosclerotic changes. The spleen weighed $350 \mathrm{~g}$. and had a firm purple cut surface with prominent Malpighian corpuscles. The large bowel had numerous diverticula, and there was a $1 \mathrm{~cm}$. mucosal polyp in the sigmoid portion. The kidneys weighed about $100 \mathrm{~g}$. and had a granular surface with a few broad, flat-based scars. The bone marrow of the vertebrae was red and moist with scattered foci of fat replacement.

There was focal interstitial fibrosis of the myocardium. Overdistending pulmonary alveoli were often filled with pale eosinophilic-staining material and macrophages laden with brown pigment. An occasional pulmonary artery contained immature myeloid cells. The splenic Malpighian corpuscles were active and spread apart by expanded red pulp sinusoids filled with red cells and immature myeloids. Adrenal cortices were depleted of lipid. The kidneys showed changes of diffuse arteriolar nephrosclerosis and healed pyelonephritis. The lymph nodes from various sites appeared inactive, but peripheral sinuses often contained immature myeloids. The vertebral bone marrow was hypercellular with immature myeloids and blast forms predominating. But groups of red cell precursors and megakaryocytes were seen in most fields. It was a very similar picture to that seen in the antemortem specimen, and it was felt that these findings represented a myeloproliferative disorder, consistent with acute myelogenous leukemia.

Neuropathological findings The brain weighed $1,280 \mathrm{~g}$. at necropsy. The dura on its inner surface displayed small patchy collections of recent haemorrhage, up to $3 \mathrm{~mm}$. in thickness. There was slight to moderate atherosclerosis of the arteries of the circle of Willis. The external configuration of the brain was normal except for a slight generalized widening of sulci and narrowing of gyri, most marked at the frontal poles. Coronal sections revealed mild dilatation of the lateral ventricles. The brain parenchyma was pale. No focal lesions were noted.

Routine paraffin sections stained with haematoxylin and eosin revealed marked senile changes with numerous senile plaques in all sections of the cortex. A few examples of neurofibrillary changes were seen in the innominate substance, the hypothalamus, the substantia nigra, locus caeruleus, and oculomotor nucleus; and they were later demonstrated in great numbers in the cortex with silver stains on frozen sections. In the cerebral cortex, there was slight to moderate nerve cell loss, scattered sclerotic nerve cells, and in some areas a mild increase in astrocytes. Two 1-2 mm. areas of recent necrosis, with palestaining vacuolated tissue and nerve cell loss, were also present in the cerebral cortex. Ferrugination of vessels was present in the globus pallidus and in the Ammon's 
horn. There was a slight loss of nerve cells in the pigmented nuclei of the brain-stem. The cerebellar cortex displayed a slight, diffuse reduction in the number of Purkinje cells. There was a moderate degree of thickening with hyalinization of the small arteries and arterioles.

The most interesting finding, however, was the presence of several small focal lesions in the white matter and junction of cortex and white matter in two sections from the left frontal lobe (Figs. 1 and 2). These lesions presented as pale, rounded, or oval areas surrounded by a more cellular border of intensely basophilic nuclei, reacting astrocytes, and elongated microglia. The centre usually contained a few astrocytes and a normal or slightly reduced number of oligodendrocytes. The lesions measured from $100 \mu$ to $1 \mathrm{~mm}$. in maximal diameter. The basophilic nuclei measured from 10 to $14 \mu$ in average diameter. They were often surrounded by a small amount of cytoplasm. Some of them were circular, but others had a triangular shape, or had irregular outlines, often with scalloped margins. The internal structure of the nuclei was frequently completely lost. No typical Cowdry type A intranuclear inclusions were seen, but some nuclei had a mauve tint and others displayed a slight margination of the chromatin. No giant astrocytes were present, and no mitotic figures were seen in any of the cells. While small capillaries and occasionally veins were sometimes seen within the lesions, there did not seem to be any constant relation

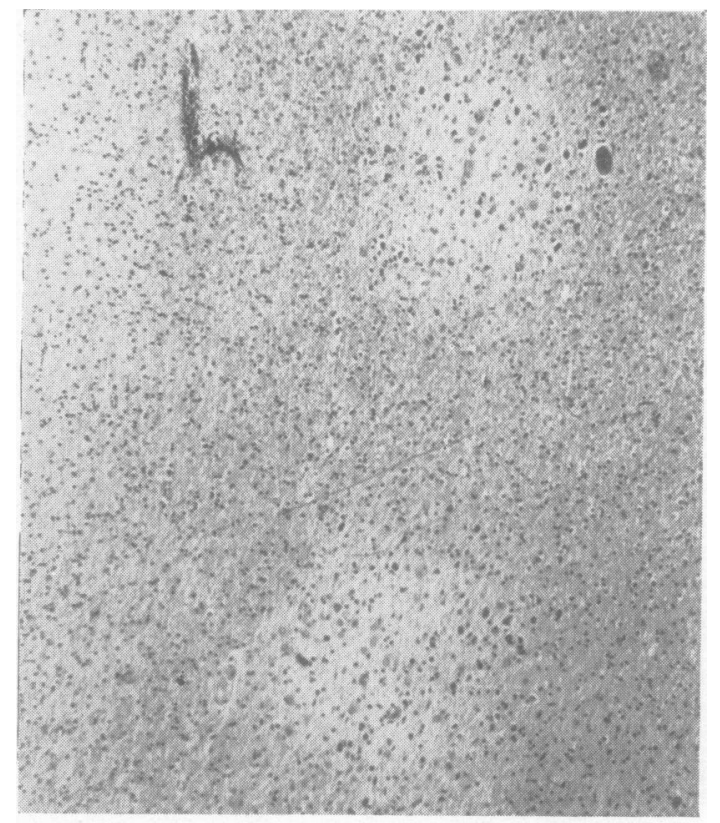

FIG. 1 . to vessels. Some veins and capillaries were filled with leukaemic cells, but no leukaemic infiltration was present in the brain parenchyma. Perivenous lymphocytic cuffing was minimal.

A search for more lesions of this nature was made with additional paraffin blocks and a set of representative celloidin sections. More small lesions were found in the white matter of both cerebral hemispheres, especially in the vicinity of the sensorimotor cortex bilaterally and in the cerebellum. The most numerous lesions were present in the parasagittal region of the left frontal lobe. The lesions seen here in celloidin sections were generally slightly larger than the ones first seen and measured up to $2 \mathrm{~mm}$. All lesions in the celloidin sections showed a marked proliferation of pleomorphic and elongated microglia as well as many reacting astrocytes within the lesion. Again no giant cells were present. The peripheral basophilic nuclei were best seen in the sections stained for myelin (Loyez modification) (Fig. 3).

The lesions in the cerebellum were less typical than the ones in the centrum semiovale in that peripheral abnormal basophilic nuclei were seldom seen (Fig. 4). Also, there was often a more diffuse thinning of myelin with reactive fibrous gliosis in the white matter of the folia in addition to the well-defined focal lesions. The latter were usually situated at the junction of the cerebellar cortex with white matter and frequently extendedo

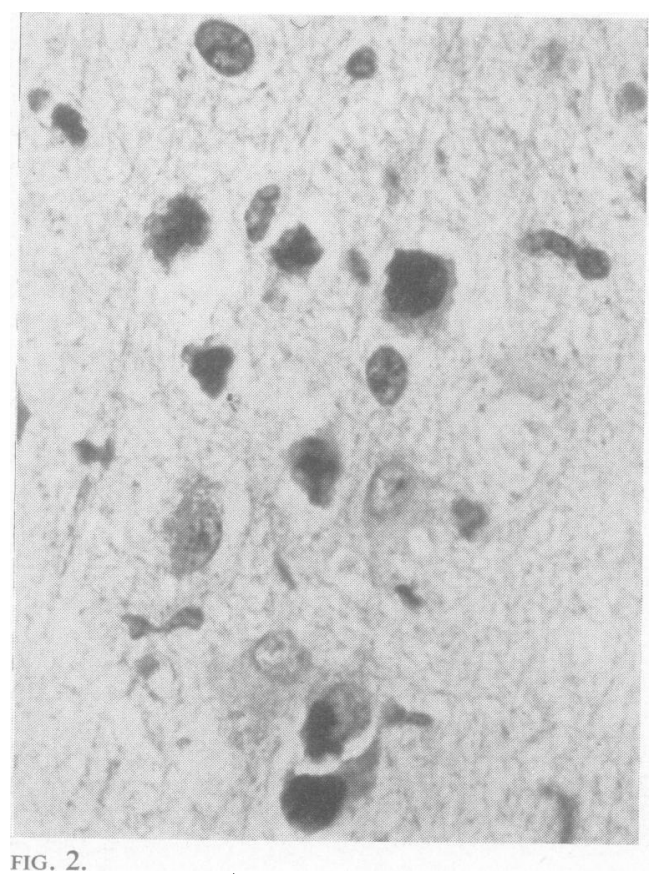

FIG. 2.

FIG. 1. Case 1 (A.M.). Subcortical white matter of left frontal lobe. Paraffin, haematoxylin and eosin $\times 70.7$ ino typical lesions of progressive multifocal leucoencephalopathy with peripheral rim of altered oligodendroglia.

FIG. 2. Case 1 (A.M.). Subcortical white matter of left frontal lobe. Paraffin, haematoxylin and eosin $\times 600$. Periphery of progressive multifocal leucoencephalopathy lesions with abnormal oligodendroglia, reacting astrocytes, and microglia. 


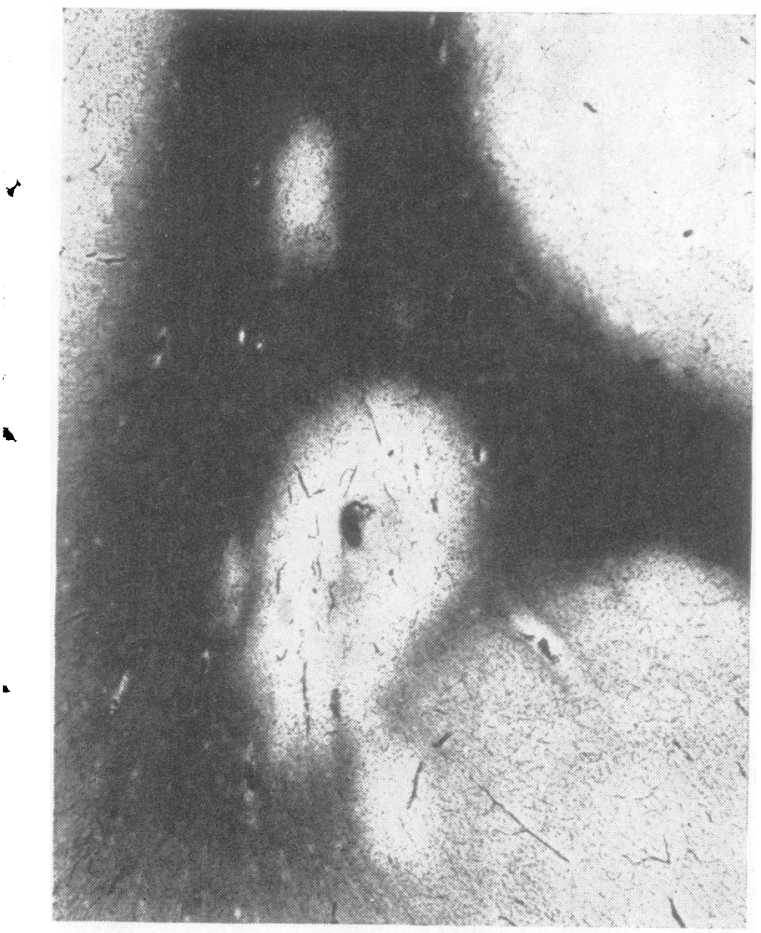

FIG. 3 .

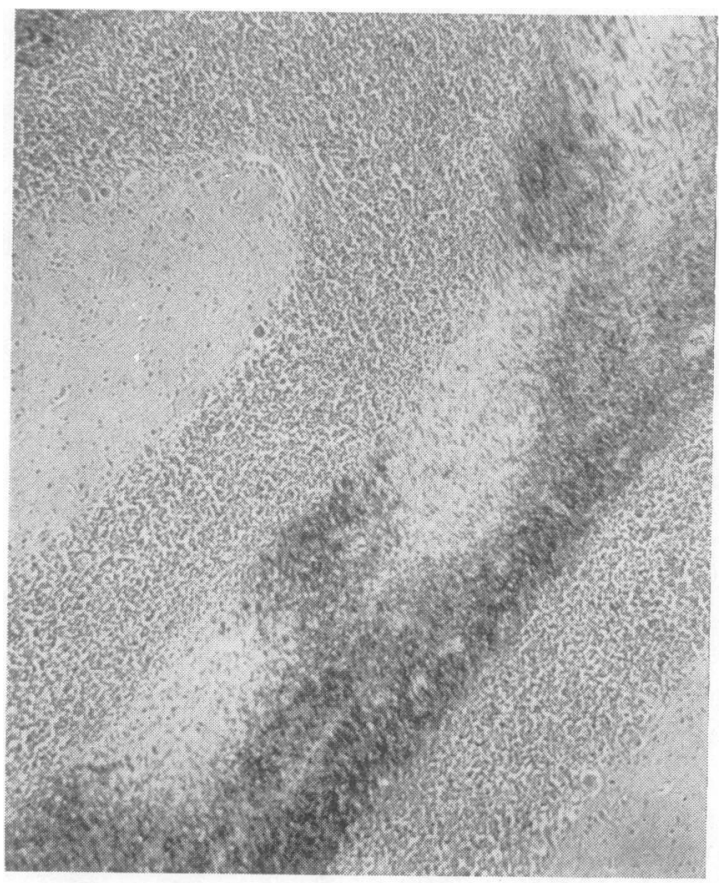

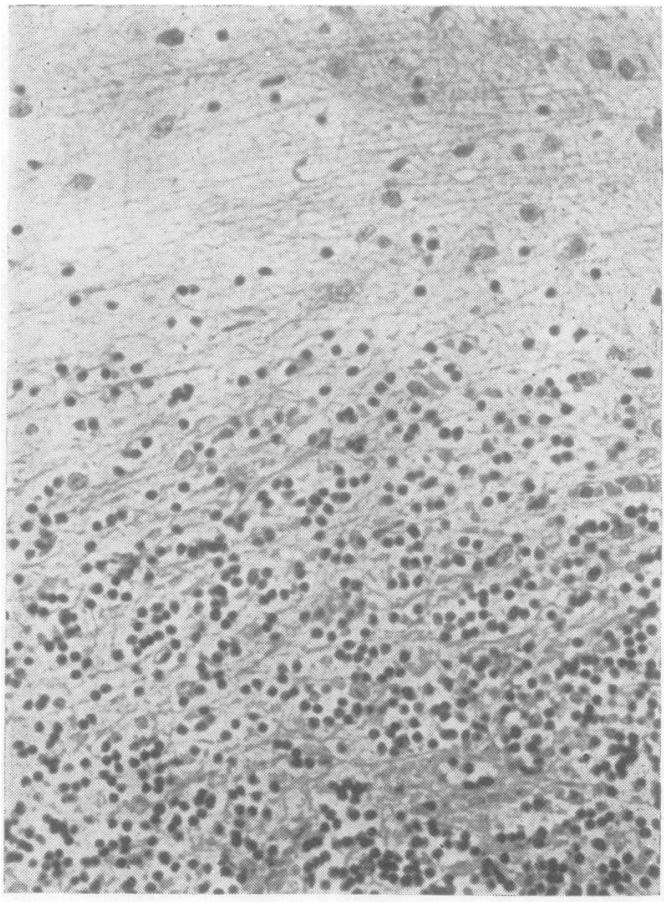

FIG. 5.

FIG. 3. Case 1 (A.M.). Left frontal lobe. Celloidin. Myelin stain (Loyez). $\times 23$. Note the peripheral rim of dark around the nuclei, extending into areas with partial demyelination. The perivascular distribution seen in the largest lesion is an exception rather than the rule. The photograph shows the largest lesions demonstrated in this case. The minute pale staining areas in the deep cortex correspond to the distribution of senile plaques.

FIG. 4. Case 1 (A.M.). Cerebellum. Paraffin. Myelin (iron-haematoxylin). $\times 60$. Two focal lesions in the white matter of the folia, encroaching on the granular layer, seen in addition to less well-defined loss of myelinated fibres.

FIG. 5. Case 1 (A.M.). Cerebellum. Junction of granular layer and white matter. Paraffin, haematoxylin and eosin $\times$ 235. Gliosis in white matter; thinning of granular cells; scattered, slightly larger and paler nuclei in granular layer (see text). 
into the granular layer. When this occurred, there was thinning of the granule cells and an increase of somewhat larger, stippled nuclei, resembling astrocyte nuclei (Fig. 5). The two lesions seen in the subcortical white matter near the right sensorimotor cortex also lacked the abnormal basophilic nuclei.

The original paraffin block displaying the largest number of lesions was used for special studies. Myelin stains (Loyez) displayed loss of myelin corresponding to the areas of cellular abnormality, but with somewhat ill-defined margins. Myelin degeneration was not complete in the smallest lesions. In the myelin stains on celloidin sections a wide border of enlarged oligodendroglial cells was present in the area of partial demyelination along the periphery. Silver stains for axis cylinders revealed a better preservation of axis cylinders than of myelin sheaths, with only a very slight reduction in the number of fibres. The presence of glial fibres was well demonstrated in P.T.A.H. and Gomori's trichrome stains. The P.A.S. reaction, phloxine-methylene-blue, and Lendrum's phloxine tartrazine stains did not yield any new information. Fifty serial sections were cut from this paraffin block, and in addition to the special stains 40 haematoxylin-and-eosin-stained serial sections were studied in order to learn about the relation of the lesions to vessels and to see all levels of the lesions. No constant relation to vessels was found. Samples of frozen sections did not yield any of the characteristic lesions, and in Bielschowsky preparations only numerous examples of neurofibrillary changes in nerve cells in the cortex and senile plaques were demonstrated.

CASE 2 A.C., a 70-year-old Caucasian male poultry labourer, was admitted to the Palo Alto Veterans Administration Hospital on 11 November 1962, because of pain in the left side of the throat and dysphagia of five days' duration. Two months before admission the patient noted small, painless lumps on the left side of his neck (which allegedly did not increase in size). There was no change in his state until the occurrence of cervical pain which prompted him to seek medical consultation. His past medical history contributed nothing.

Physical examination revealed a thin, chronically ill white male. Both palatine tonsils were enlarged, the left extending to the midline. The right tonsil had a smooth surface, but there were superficial ulcerations on the left. There were numerous painless, movable lymph nodes on both sides of the neck, which ranged from 0.5 to $2 \mathrm{~cm}$. in maximum dimension. Similar nodes were present in both right and left axillary and inguinal areas. The liver border was palpable $8 \mathrm{~cm}$. below the right costal margin, and the spleen was palpable $12 \mathrm{~cm}$. below the left costal margin. The neurological examination was unremarkable.

Laboratory studies included a haemoglobin of 6.4 g. $/ 100 \mathrm{ml}$., haematocrit of $21 \%$, white blood cell count of $164,400 /$ c.mm., with a differential of $96 \%$ lymphocytes and $4 \%$ neutrophils, platelet count of $252,000 /$ c.mm., and $4.9 \%$ reticulocytes. The sedimentation rate was 117 (Westergren). Analysis of urine revealed rare blood cells and occasional white blood cells per high-power field. The blood urea nitrogen was $26 \mathrm{mg} . / 100 \mathrm{ml}$., and the fasting blood sugar was $88 \mathrm{mg} . / 100 \mathrm{ml}$. Liver function studies showed a bilirubin of $0.82 \mathrm{mg} . / 100 \mathrm{ml}$., thymol turbidity 4.4 units, total protein $7.58 \mathrm{~g} . / 100 \mathrm{ml}$. with $3.75 \mathrm{~g}$. albumin and $3.83 \mathrm{~g}$. globulin, alkaline phosphatase, 32 Bodansky units, serum glutamic oxalocetic transaminase 28 units, and prothrombin time $74.8 \%$. The uric acid was $7.8 \mathrm{mg} . / 100 \mathrm{ml}$. Serum electrophoresis showed albumin $41 \%$, alpha I globulin $6 \%$, alpha II $14 \%$, beta $15 \%$, gamma $22 \%$. Bone marrow examination revealed a hypercellular marrow which consisted mainly of small lymphocytes, rare myeloid cells, rare megakaryocytes, and no recognizable erythroid cells.

The patient was treated with Prednisone, $20 \mathrm{mg}$. Fer day for approximately 18 weeks, and was given a total of 250 rads of $x$-irradiation to the tonsillar areas and a total of 250 rads to the spleen. While on Prednisone he was given isonicotinic acid hydrazide, $300 \mathrm{mg}$. per day.

As a result of this treatment, there was a marked decrease in tonsillar size, a minimal decrease in splenic size, and symptomatic relief from the cervical pain and dysphagia. However, the white blood cell count did not fall below $116,000 / \mathrm{c} . \mathrm{mm}$., and platelet count dropped to $88,000 /$ c.mm.

The patient was followed up in the haematology clinic, and on 15 February 1963, he was given Chlorambucil, $6 \mathrm{mg}$. per day, because of increasing generalized lymphadenopathy and hepatosplenomegaly.

On 8 March 1963, he was again admitted to the hospital because of dysphagia, fever, and productive cough. $\bar{O}$ Again marked tonsillar enlargement was noted, and there $\overrightarrow{\mathbb{D}}$ was little change in the size of liver, spleen, and lymph $\frac{\text { }}{\mathrm{Q}}$ nodes as compared with that on the initial admission. 으 There were bilateral inspiratory rales with decreased breath sounds and decreased tactile fremitus over then right lower lung field posteriorly.

Both the throat and urine cultures grew colonies of $\beta=$ haemolytic streptococci, and the chest radiograph revealed infiltrates compatible with bilateral lower lobe pneumonitis.

The patient was treated with penicillin and received 1,800 rads in 18 days to the area of Waldeyer's ring. The pneumonia and pharyngitis responded to antibiotic therapy, and there was a $70 \%$ reduction of lymphoid tissue in the tonsillar area. Because of a haematocrit of $30 \%$, he was given $500 \mathrm{ml}$. of packed red blood cells. At the time of discharge on 29 March 1963, he was begun on a regimen of cyclophosphamide, $100 \mathrm{mg}$. per day.

After 70 days' treatment with cyclophosphamide alone his condition deteriorated, with generalized lymph node enlargement and peripheral oedema of both legs, presumably due to extrinsic pressure exerted by masses of inguinal and para-aortic lymph nodes.

On 14 June 1963, he was admitted to the hospital for further treatment. Cyclophosphamide was discontinued and he was started on a course of Vincristin, $0.025 \mathrm{mg} . / \mathrm{kg}$. weekly. On this drug there was some regression in size of lymph nodes and loss of anorexia. In early July he was seen by dermatologists because of a purplish papular eruption on both lower extremities, and at this time a healing herpes simplex eruption on the right lower lip was also noted. His hospital course was otherwise unremarkable until 9 August 1963, when he was noted to have fairly marked weakness of the right arm. Neuro- 
logical examination, three days later revealed decreased strength in the right arm, mild generalized weakness, ataxia which was not explained by the weakness, right infraspinatus atrophy, and an extensor plantar reflex on the right. The left biceps reflex was more active than the right. There was no sensory deficit.

On 13 August the patient fell, striking the back of his head, but he did not lose consciousness, and the neurological findings were essentially unchanged. The next morning his temperature was $39 \cdot 3^{\circ} \mathrm{C}$., pulse 140 and regular, respiration 20 , blood pressure $115 / 70 \mathrm{~mm}$. $\mathbf{H g}$, and he complained of cough. Examination showed dry mucous membranes, poor skin turgor, and dullness with decreased breath sounds and coarse rales over the right lower lobe of the lung. There was asterixis and weakness of the right shoulder, arm, and leg. The face and hand were not involved on the right. The right plantar reflex was flexor. On penicillin he became afebrile, and within a week there was increase in strength on the right and asterixis was decreasing. The spinal fluid was under opening pressure of 210 and contained 2 red blood cells per c.mm., and no white blood cells. The cerebrospinal fluid protein was $36 \mathrm{mg}$. $/ 100 \mathrm{ml}$. and sugar $61 \mathrm{mg} . / 100 \mathrm{ml}$. with a simultaneous blood sugar of $180 \mathrm{mg} . / 100 \mathrm{ml}$. The electroencephalogram (E.E.G.) on 14 August showed a waking record with irregular symmetrical low voltage and 6 to 8 per second slow alpha activity in the posterior hemisphere. At times, slower irregular frequency in the 4 to 5 per second range was more evident. Occasionally, irregular delta and theta activity and sharp and slow waves were seen in the posterior hemispheres, more prominently in the left posterior temporal area. The neurological opinion at this time was that the patient had a lesion involving the left hemisphere in the motor areas of the right leg, shoulder, and arm, but sparing the face and hand. During the following weeks, there was a slow progression of the patient's neurological symptoms, and on 7 September a right central facial nerve weakness and a weak right sternomastoid were noted.

On 18 September the neurological examination revealed a cooperative, pleasant man who performed tasks slowly and displayed pathological lability and poor memory for remote events. The main findings were right central facial weakness, right flaccid hemiparesis, and a positive snout reflex with some sucking component. The visual fields were normal. A repeat E.E.G. revealed slow wave activity in the 5 to 6 -second per cycle range, more prominent in the left posterior region. This record was very similar and only slightly more abnormal than the one taken a month earlier. Lumbar puncture on 10 September showed no cells, protein of $66.2 \mathrm{mg} . / 100 \mathrm{ml}$., and sugar $63 \mathrm{mg} . / 100 \mathrm{ml}$. Because of a remaining suspicion that leukaemic infiltrates might be responsible for the neurological picture, he was given a total of 1,500 rads of whole brain irradiation over a period of three weeks. No demonstrable improvement occurred from this or from the continued Vincristin therapy. The patient's neurological illness progressed and aphasia developed about 1 November. Asterixis continued to be present on an intermittent basis. The terminal course was rapid and marked by severe anaemia requiring multiple blood transfusions and by staphylococcal pneumonia. He terminally lapsed into coma and expired on 1 December 1963, scarcely four months after the onset of neurological symptoms and 14 months after the symptoms of leukaemia first appeared.

Necropsy The post-mortem examination (PAVA 63-A-198) was performed 30 hours after death. The body was that of an emaciated elderly male whose appearance was consistent with the stated age, 71 years.

There was generalized lymphadenopathy involving cervical, axillary, mediastinal, mesenteric, femoral, and inguinal groups of nodes, with dense tumour infiltrates and loss of normal architecture seen on microscopic sections.

Both the liver and spleen were enlarged, with massive tumour infiltrates in both organs. Characteristically, the tumour cells were in the portal areas of the liver.

The lungs revealed both tumour infiltrates and acute bronchopneumonia. The bone marrow showed almost complete replacement with tumour.

Neuropathological findings The brain weighed $1,560 \mathrm{~g}$. at necropsy. There was only very slight atherosclerosis of the arteries of the circle of Willis. There was a soft, bulging area $5 \mathrm{~cm}$. in diameter over the upper convexity on the left, and a slight prominence of the corresponding area on the right. No marked flattening of the gyri or narrowing of the sulci were present, but a small pressure cone was seen on the left uncus and a grooving on the right. There were no cerebellar pressure cones. Coronal sections after fixation revealed a spectacular, predominantly white matter lesion in the upper left cerebral hemisphere, with a number of smaller lesions along the margin, where they extended for a varying distance into the cerebral cortex (Fig. 6). On the right a number of small, 1 to $4 \mathrm{~mm}$., lesions were seen at the junction of the cortex and white matter, most numerous in the upper convexity. The large left hemisphere lesion measured 5 by $4 \mathrm{~cm}$. in largest diameter in the coronal plane and extended from the level of the optic chiasm to $3 \mathrm{~cm}$. beyond the splenium. It was gray, granular, and quite soft. The smaller lesions were often of a more homogenous translucency. The large softening had its greatest dimensions posteriorly, and here the most marked involvement of the cortex occurred. The corpus callosum was affected in its left half at the level

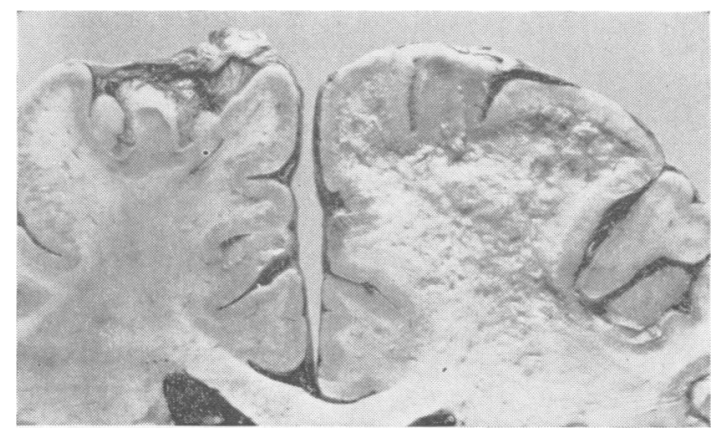

FIG. 6. Case 2 (A.C.). Coronal section after formalin fixation, showing the large lesion in the left cerebral hemisphere and multiple small lesions at junction of cortex and white matter on the right, at the level of the pineal gland. 
of the subthalamic nucleus. On the right the most numerous lesions were present at the level of the pineal gland, but scattered lesions were also present through the hemispheres.

Several scattered and confluent lesions were seen in the left lower claustrum and in the upper portion of the white matter of the temporal lobe at the level of the chiasm. The basal ganglia did not show gross lesions except in the lateral left putamen. The thalami were affected with mottled gray, translucent lesions at the level of the splenium, most marked on the left. The brain-stem displayed a few tiny, mottled, translucent areas. The cerebellum likewise revealed small lesions. The ventricular system was within normal limits. Only the upper cervical cord was available for study.

The brain was examined in paraffin and celloidin embedded blocks and with frozen section techniques. The paraffin sections were stained routinely with haematoxylin and eosin, the cellodin sections with cresyl violet (Nissl technique) and with the Loyez modification for myelin. In addition, a number of paraffin blocks were stained with myelin stains (Loyez or luxol fast blue), Gomori's trichrome stain, P.T.A.H., Holzer stain for glial fibres, periodic-acid-Schiff reaction (P.A.S. stain), Lendrum's phloxine tartrazine stain, phloxine-methyleneblue, silver impregnation for axis cylinders (Palmgren's method) and Wilder's stain for reticulum. Frozen sections were impregnated with silver (Gros-Bielschowsky modification) and with Hortega's silver carbonate for oligodendroglia and microglia. Oil red $O$ stain for fat, Sudan black b stain, and acid cresyl violet for metachromasia were also carried out on frozen sections.

The localization of many of the small lesions at the junction of the cortex and white matter was characteristic, and there was no tendency towards sparing of the Ufibres (Fig. 7). The large lesions at all levels extended into the overlying cortex on the upper convexity of the left hemisphere, and at one level the lesion reached to the first layer of the cortex. On the left, small scattered lesions were seen as far forward as the tip of the frontal lobe; however, no lesions were found in the left occipital pole. The corpus callosum was affected in the left half in the posterior portion of the body, and small lesions were pres-

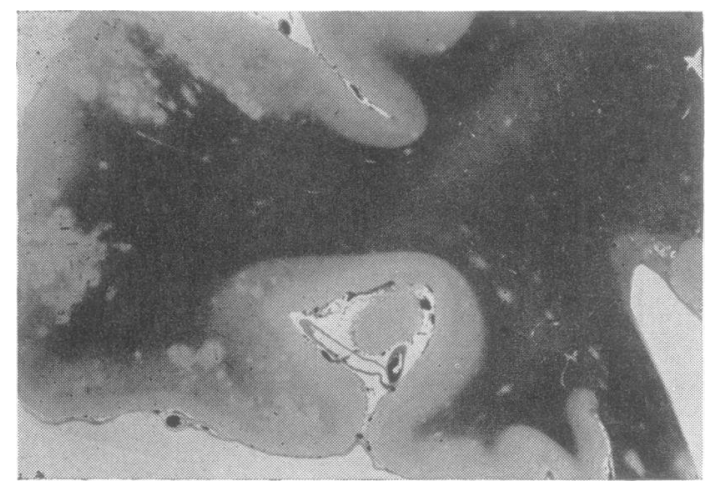

FIG. 7. Case 2 (A.C.). Right hemisphere at level of subthalmic nucleus. Celloidin. Myelin (Loyez). $\times 3$. ent in the right half at this level. The anterior commissure, fornix, and internal capsule were not involved at the levels examined.

The basal ganglia were only slightly affected except for the lateral portion of the putamen which showed a number of partially confluent lesions, especially on the left. The claustrum and adjacent portions of the external capsule displayed prominent lesions, again more on the left. The adjacent portion of the temporal lobe contained only a few small, scattered lesions, usually at the junction of the cortex and white matter. The thalamus on the right contained a few lesions, but on the left large confluent lesions were present at the level of the lateral geniculate body. The hypothalamus showed only a few small lesions.

In the brain-stem and cerebellum several small lesions were present in the cerebral peduncles, substantia nigra, basilar and tegmental pons, and in the pyramids of the medulla. In the upper cervical cord several tiny lesions were noted in the lateral white columns bilaterally. The majority of the small lesions in the cerebellum were located in the white matter, but an occasional one was seen in the granular layer or at the junction of the cerebellar cortex and white matter. A slight thinning of granule cells was noted here and large stippled nuclei were seen in these areas, similar to the findings in case 1.

The histological picture was characterized by complete or partial loss of myelinated fibres with relative sparing of axis cylinders and the now classical findings of giant $\frac{1}{1}$ astrocytes and round basophilic nuclei, resembling oligodendrocytes (Figs. 8, 9, 10,11, and 12). In the large $\varrho$ lesions a characteristic mixture of phagocytic cells and $\overrightarrow{-}$ reacting astrocytes, along with scattered giant astrocytes, $\rho$ was present. The smaller lesions usually did not contain phagocytic cells and were often less cellular, with a few reacting astrocytes and sometimes elongated and pleo-0 morphic microglia. The best examples of the abnormal? oligodendroglial nuclei were seen along the margin of these small lesions, but they were generally less prominent than in case 1 . As in that case, no completely convincing examples of intranuclear inclusions were found; some of the enlarged oligodendroglial nuclei displayed intensely staining chromatin particles, while others presented as a dark, homogenous mass which sometimes assumed a slightly reddish or purplish-red colour in haematoxylin and eosin stain. Special stains for inclusion bodies were negative. A narrow rim of pale pink cytoplasm often surrounded these nuclei.

Giant cells were not numerous at any level, but one or two were present in many of the smaller lesions, and the greatest number were seen in the affected corpus callosum. They measured up to $90 \mu$ in length with the largest dimension of the nucleus reaching $60 \mu$. The cytoplasm was homogeneously pink in the haematoxylin and eosin stain, appearing as in a large reacting astrocyte. The nucleus was usually intensely hyperchromatic and of round, or oval, or irregular shape, sometimes containing one or more pink, round, homogenous inclusions. Mitotic figures were very rare, but when seen were irregular and atypical. Some giant cells contained two or more nuclei. Special stains demonstrated fibrous processes and glial fibres with attachment to vessel walls in many of the giant cells. 


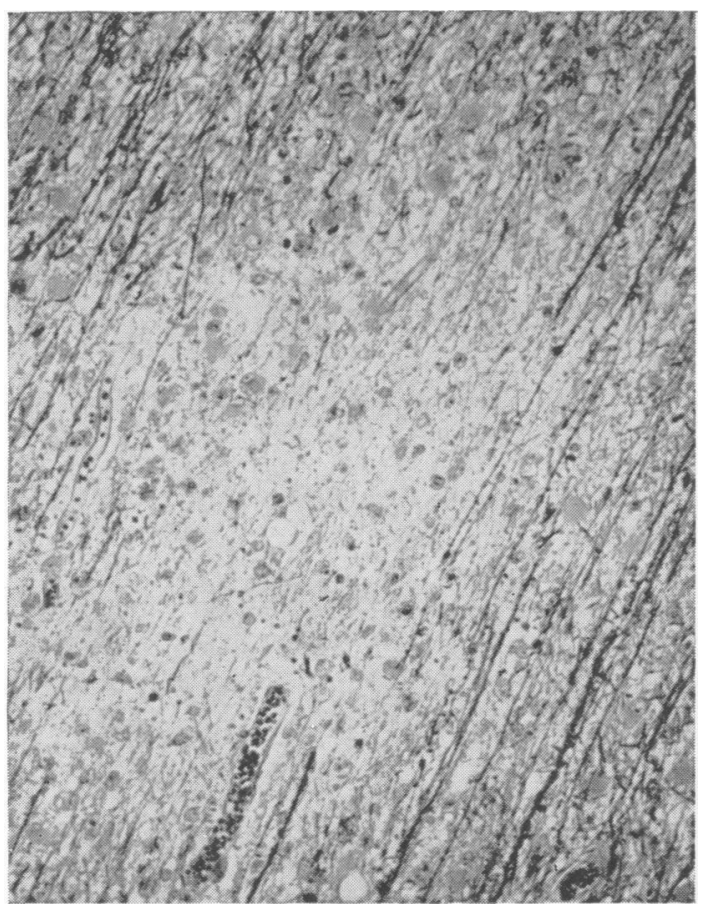

FIG. 8.

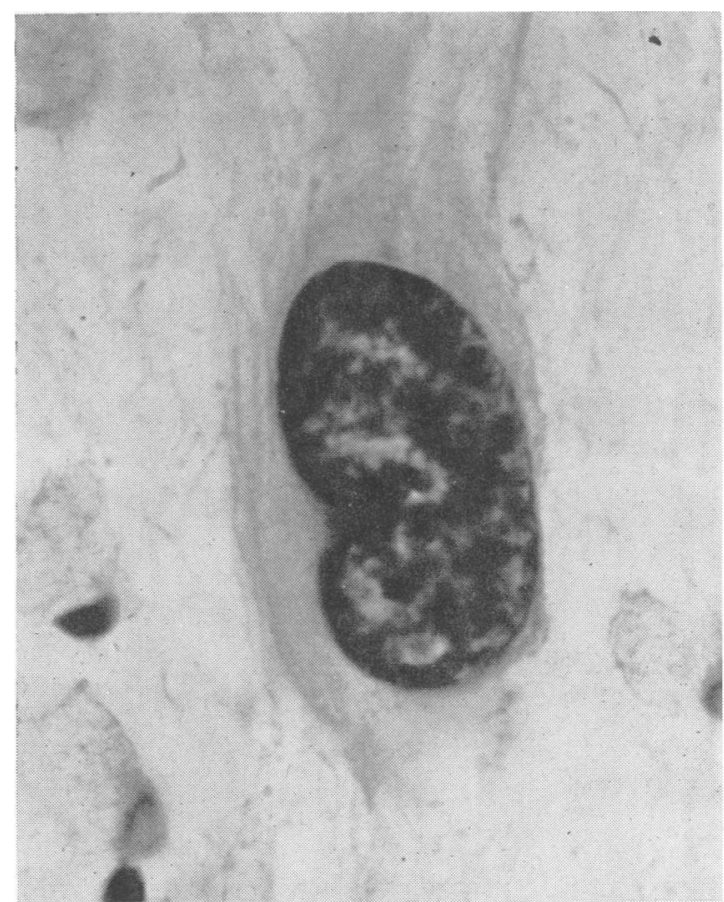

FIG. 9.

FIG. 8. Case 2 (A.C.). Paraffin. Luxol fast blue. $\times 140$. One of the small lesions in right hemisphere near the larger lesion. FIG. 9. Case 2 (A.C.). Paraffin. Haematoxylin and eosin Giant astrocyte. $\times 1,000$.

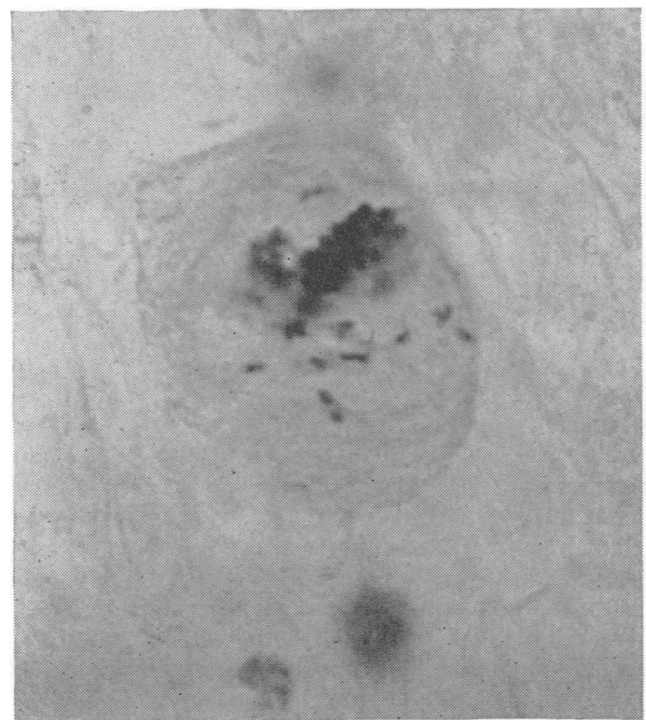

FIG. 10 .

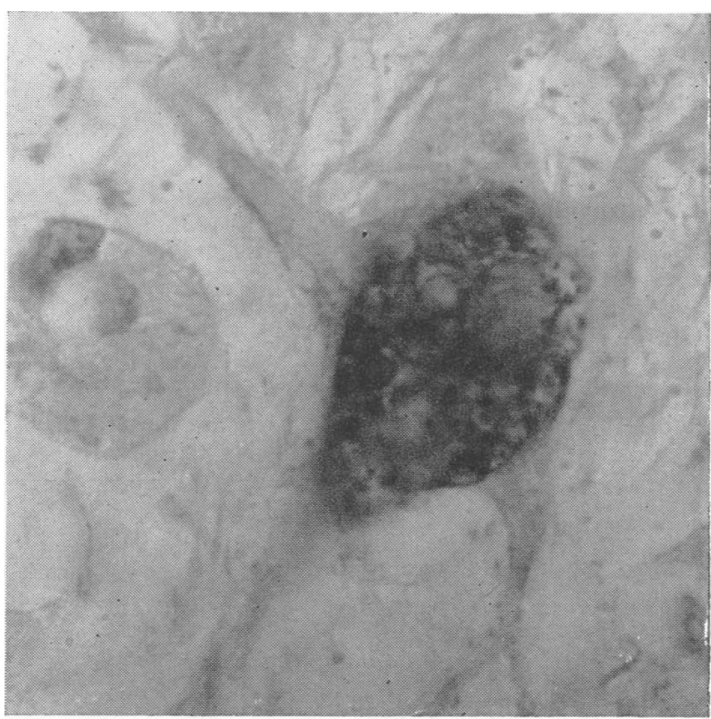

FIG. 11

FIG. 10. Case 2 (A.C.). Paraffin. Haematoxylin and eosin Atypical mitosis in giant astrocytes. $\times 1,000$.

FIG. 11. Case 2(A.C.). Paraffin. Haematoxylin and eosin $\times 1,000$. Giant astrocyte with inclusions in the nucleus. 


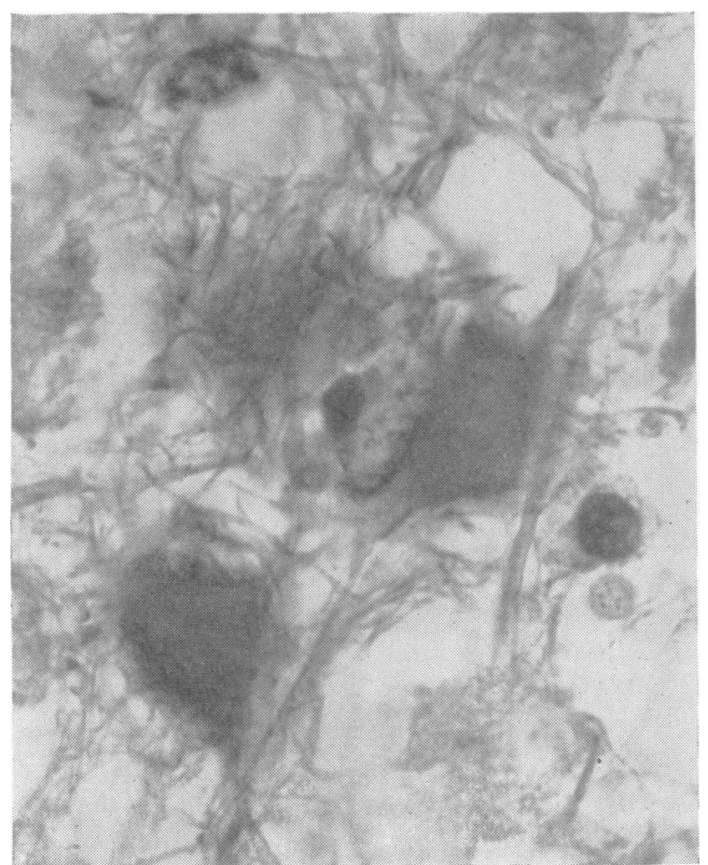

FIG. 12. Case 2 (A.C.). Paraffin. Holzer stain. $\times 1,000$. Giant astrocyte just below centre.

Fat stains revealed adundant sudanophilic material within phagocytic cells in the large lesions. In the small lesions fat was mainly present as fine granules along the periphery of reacting astrocytes in oil red $O$ stain. Acid cresyl violet stains on frozen sections, mounted in glycerin jelly, revealed no abnormal metachromasia. Periodic-acidSchiff stains on paraffin sections demonstrated the presence of numerous P.A.S.-positive granules within the cytoplasm of the phagocytic cells.

Silver impregnations for axis cylinders (Palmgren's method on paraffin, Gros-Bielschowsky on frozen sections) showed some preservation of axis cylinders, even within the large softening. But the remaining axonrs frequently showed globular swellings. Stains for myelin sheaths revealed complete loss of myelinated fibres in the larger lesions. In the smaller lesions and along the border of the larger ones a zone of partial demyelination was present. Oligodendroglial cells were often identified in these areas as enlarged, round black nuclei in this border area.

Where the lesions involved gray matter, nerve cells were well preserved, with the exception of some examples of swelling, central chromatolysis, and eccentricity of the nucleus in nerve cells in the cortex, best seen in the Betz cells. The lesions in the cerebellum resembled those seen in the rest of the central nervous system, except for the previously mentioned thinning of granule cells. This was seen mainly in the deep granular layer, and was sometimes associated with a lesion in the white matter of the folia.

In addition to the lesions characteristic of progrcssive multifocal leucoencephalopathy, defined by Åström et al. (1958) and Richardson (1961), another feature was prominent in this case, namely, heavy lymphocytic infiltration. This cellular infiltration was associated with severe leukostasis of vessels and was present in the perivascular spaces, most marked around veins, in the subarachnoid space, and in the brain parenchyma. Here the lymphocytes seemed to spill over into the tissue from the capillaries and from the perivenous cuffs. The cellular infiltrates were present throughout the brain, and were often quite marked within or adjacent to lesions (Fig. 13).

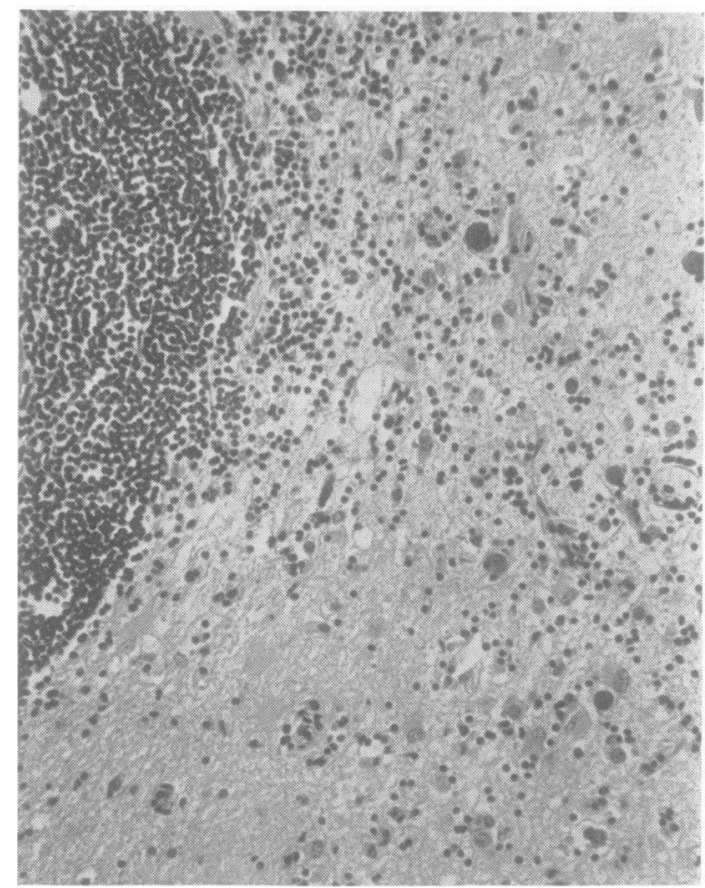

FIG. 13. Case 2 (A.C.). Paraffin. Haematoxylin and eosin $\times$ 175.Left lateral putamen. Perivenous lymphocytic cuffing and leukaemic infiltration of brain parenchyma. Reacting astrocytes and morular cells are present; the latter stain black or dark grey in the photomicrograph.

However, cellular infiltration of the tissue was also seen without any concomitant damage to the parenchyma. The lesions of progressive multifocal leucoencephalopathy were not infrequently seen surrounding a vein. Yet vessels were so prominent everywhere that this seemed to be a chance relationship, and serial sections of one paraffin block confirmed this interpretation.

In a few sections from the vicinity of the posterior left putamen, claustrum, and external capsule, there were plasma cells among the lymphocytes in the cuffs and in the parenchyma. Progressive multifocal leucoencephalopathy lesions were present in clusters in this area and they also contained lymphocytic infiltration. In one of these sections, there were in addition a number of cells, 15 to $35 \mu$ in diameter, with the appearance of morular cells. 


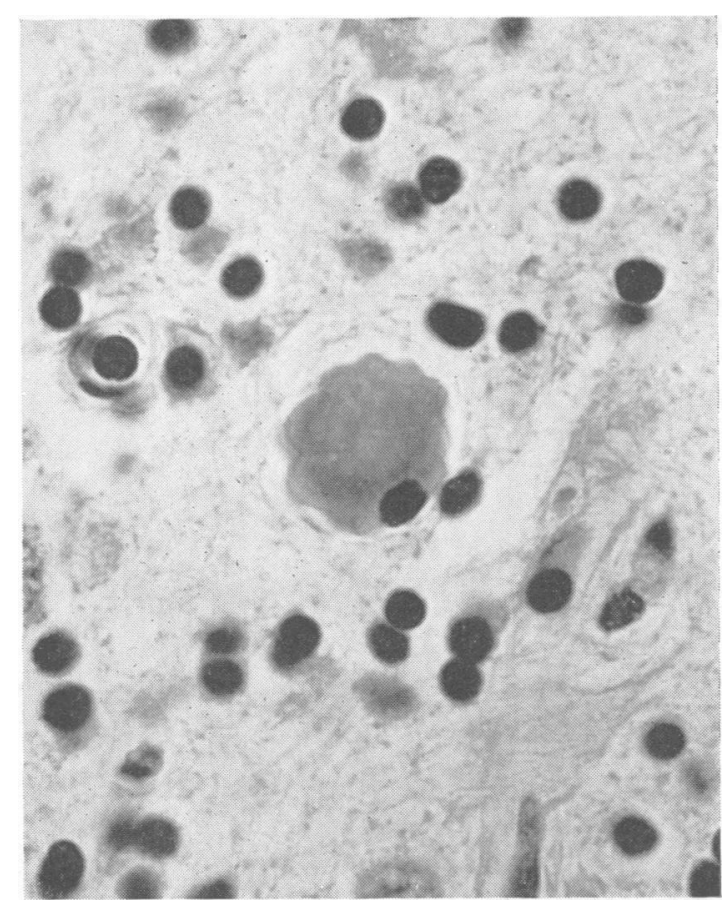

FIG. 14. Case 2 (A.C.). Paraffin. Haematoxylin and eosin $\times 1,000$. High-power view of one of the morular cells in Figure 13.

Although these cells occasionally were encountered in other areas of the brain, where progressive multifocal leucoencephalopathy lesions were found, they were nowhere as numerous as in the lateral left putamen. They were rounded or mulberry-shaped cells with a small, dark, eccentric nucleus, and cytoplasm filled with eosinophilic globular structures. They stained a dusky red in haematoxylin and eosin stain, purple to red in P.T.A.H. stain, and blue in Holzer stain. In Wilder's reticulum stain the globular structures were surrounded by and separated by a black-staining material, while the globules themselves stained only with the nuclear fast red counterstain. They were brilliantly red in Masson's and Gomori's trichrome stain. With thioflavin stain they showed marked fluorescence; they were P.A.S.-positive, Feulgen-negative, and with methyl green-Pyronin they stained purple. Sudan black B stain on paraffin section was negative for fat. In Nissl stain they varied from pale to deep blue (Fig. 14).

No other pathology was demonstrated in the sections. Amyloid bodies were present in small to moderate amounts and were occasionally seen within the progressive multifocal leucoencephalopathy lesions. No senile plaques or neurofibrillary changes were demonstrated in the special stains. There was no marked arteriosclerotic change in the vessels. No haemorrhages were present.

\section{DISCUSSION}

With the present report, approximately 30 cases of progressive multifocal leucoencephalopathy have been published; six more have been mentioned in the reports of Richardson (1961), Weinstein, Woolf, and Meynell (1963), and Deep, Fraumeni, Tashima, and McDivitt (1964), and Rubinstein(1964) has reviewed at least two cases. Although the disease may now be suspected during life, the diagnosis can only be made with certainty on biopsy or necropsy material; and the former method, as well as electron microscope studies, yet awaits trial. According to Richardson (1961), who helped to give the condition its name and whose experience with the pathology of the disease is unexcelled, the "combination of multiple demyelinative lesions of all possible gradations in size and stage of evolution, with remarkable changes in oligodendrocytes, and often in astrocytes' is pathognomonic for the disease. All of these characteristics were present in case 2 , while in case 1 only small lesions with typical abnormal oligodendroglial cells were present. The diagnosis in both cases was confirmed by Richardson.

Clinically case 2 fits in well with the picture of progressive multifocal leucoencephalopathy which has emerged from the reports in the literature. The development of a progressive neurological disorder, with hemiplegia and later aphasia, in a man with chronic lymphatic leukaemia is fairly characteristic for this disease. The essentially normal spinal fluid, normal brain scan, and the E.E.G. abnormalities also fit into the clinical picture of progressive multifocal leucoencephalopathy. Case 1 presented no such typical symptoms; at 84 , the woman is the oldest individual yet recorded with the disorder. She is also the only one with an acute myelogenous disease. The symptoms she displayed over the last three or four years of her life were related probably to senile brain damage and possibly to vascular insults. She did have some symptoms which may have been of cerebellar origin (tremor, incoordination), but these could be explained on the basis of the slight Purkinje cell loss, senile plaques in the granule layer of the cerebellar vermis, and the slight thinning of myelinated fibres in the white matter of the cerebellar folia so that the presence of progressive multifocal leucoencephalopathy did not have to be invoked.

The unique neuropathological features deserve some comments in relation to the aetiological considerations. In 1961, Richardson, supported by Waksman and Adams (1962a, b), put forward the hypothesis that progressive multifocal leucoencephalopathy is a viral disease which affects individuals with deficient immune response due to their primary disease, most commonly a disorder of the reticuloendothelial system, i.e., lymphatic leukaemia or Hodgkin's disease. Since then the viral theory has received the most support and discussion, although 
other possibilities have been mentioned. These include an auto-immune reaction (Headington and Umiker, 1962; Deep et al., 1964), and excess activity of the reticulo-endothelial system (Weinstein et al., 1963). Cavanagh, Greenbaum, Marshall, and Rubinstein (1959) also suggested a disturbance involving lipid metabolism, in particular that of sphingomyelin.

In our opinion a viral infection offers the best explanation of the pathology. Certain viral infections in animals, e.g., moose encephalitis, and under certain circumstances, herpes simplex encephalitis in rabbits, are capable of producing similar lesions with spotty loss of myelin, predominantly in the white matter (Waksman and Adams, 1962a, b). These lesions differ in important respects from the pathology seen in experimental allergic encephalomyelitis, disseminated encephalomyelitis of post-infectious or post-exanthematous type in humans, and multiple sclerosis. In experimental allergic encephalomyelitis perivascular cellular infiltration is probably the initial event and appears to cause the myelin breakdown. This was shown (Waksman and Adams, 1962a, b) in guinea pigs and rabbits. Our own experience with experimental allergic encephalomyelitis lesions in rats (Forno and Smith, 1964, unpublished data) seems to support this. In the viral diseases and in progressive multiple leucoencephalomyelitis, the white matter appears primarily affected, sometimes with astrocytes and oligodendroglial cells bearing the brunt of the change. This is well exemplified in case 1 . All the lesions here appeared to be in a relatively early stage. Perivascular cuffing was insignificant, and there was no constant relation of the lesions to vessels. Especially characteristic was the large number of abnormal oligodendroglial cells in the partially demyelinated area along the margin of the lesions. In the second case the small lesions had a similar appearance.

The very distinctive abnormalities in oligodendrocytes and astrocytes are other points favouring a virus infection. It is agreed by most authors (D'Agostino, Pease, and Kernohan, 1963) that the round, basophilic nuclei along the periphery of the progressive multifocal leucoencephalomyelitis lesions represent enlarged nuclei of oligodendroglia. Weinstein et al. (1963) interpreted these structures as extruded nuclei from giant cells. This view would be difficult as a general one, especially in such cases as case 1 and in several of Richardson's (1961) cases where giant cells were not present. In case 2 , silver carbonate impregnation of oligodendroglia was tried but without any conclusive results; impregnation of the cellular processes was imperfect both in the lesions and in normal white matter. The abnormal nuclei were intensely stained in silver carbonate as well as in
Gros-Bielschowsky preparations, but in the latter they were sometimes easily confused with globular axonal swellings. The fate of the oligodendroglial cells within an area of demyelination is always of some interest. In both our cases, there was often slight reduction in the number of oligodendroglial cells, but never a complete loss. Even in the largest lesions some oligodendroglia appeared to be present, although often difficult to distinguish from small microglial cells and lymphocytes.

In our two cases distinct Cowdry type $\mathbf{A}$ intranuclear inclusions were not demonstrated in the abnormal oligodendroglial cells, but they have been visualized in the majority of cases reported. It appears likely that some of the changes we observed, e.g., the shrinkage of the nucleoprotein away from the nuclear membrane, the homogenization of the chromatin, and the mauve discoloration, may represent stages in the formation of inclusions. They are indeed quite similar to stages in the development of inclusions described by Bird, Ennis, Wort, and Gardner (1963) in the hepatic nuclei in newborn infants with disseminated herpes simplex.

Giant cells were present in case 2 only. They were 0 seen in most lesions, except the smallest, but were never very numerous. The nuclei usually stained intensely and were often irregular in shape and some $\frac{\mathbb{D}}{2}$ 음 times lobulated. Two or more nuclei might be found $\vec{b}$ in one cell. Round, pale inclusions were present in

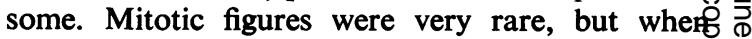
present were irregular and atypical. Weinstein et al $\vec{\oplus}$ (1963) have expressed some doubt in regard to the astrocytic nature of these cells because of the stain:ing of their processes with stains for reticulum and lack of impregnation with P.T.A.H. stain. We have no such misgivings in our case. Glial fibres were demonstrated in P.T.A.H., trichrome, and Holzer stains, although not all giant cells showed as welldeveloped glial fibres as the reacting astrocytes. In Wilder's reticulum stain, giant astrocytes as well as reacting astrocytes were often impregnated, but the processes showed the characteristic attachment to vessel walls, and Bielschowsky and Palmgren silver impregnation showed a similar picture.

The changes seen in the granular layer of the cerebellum in both cases were similar but not very conspicuous; we are not sure that they correspond to the changes described by Richardson (1961). The large stippled nuclei may represent astrocytes and not altered granule cells. Some of these nuclei may belong to the large Golgi type II nerve cells normally present in the granular layer but made more prominent by the loss of the granule cells.

The heavy lymphocytic cuffing and parenchymal infiltration with lymphocytes in case 2 give rise to some deliberations. In the majority of cases of 
progressive multifocal leucoencephalomyelitis perivascular cuffing and inflammatory reactions have been minimal, although three of Richardson's cases (1961) and that of Weinstein et al. (1963) showed more marked cuffing. We have interpreted these findings in case 2 as severe leukaemic infiltration, although we regard true leukaemic infiltration of the brain tissue, without associated haemorrhage, as relatively rare.

The presence of plasma cells in the perivascular and parenchymal infiltrates is unusual. Plasma cells were seen in only one of the cases (case 4) described by Richardson (1961). The fact that this was a heterogenous infiltrate suggests a combination of leukaemic infiltration and inflammatory reaction.

The finding of morular cells of Mott, most numerous in the left lateral putamen, where plasma cells were also most prominent, is also of some interest. The hyaline acidophilic inclusions of these cells are probably identical with the Russell bodies of plasma cells. Russell body cells and grape cells of hyperglobulinaemia as well as morular cells may represent the same special change in plasma cells (Welsh, 1962). It is quite possible that morular cells reflect only the presence of plasma cells. It may be significant, however, that they have been found mainly in inflammatory disorders. They are particularly numerous in trypanosomiasis (Mott, 1907; Janssen, Van Bogaert, and Haymaker, 1956) but have also been found in general paresis, tuberculous meningitis, experimental blastomycosis (Spielmeyer, 1922) and in severe inclusion body encephalitis (Janssen et al., 1956). We have personally seen them in a case of coccidioides meningitis. Thus their presence may lend some slight support to the infectious hypothesis.

Because of this suggestive evidence, viral studies during life in suspected cases as well as at necropsy are urgently needed, together with biochemical and histochemical studies. Early cases such as case 1 can probably not be diagnosed during life, but may be found more frequently than in the past by careful examination of routine haematoxylin-and-eosinstained sections, as the disease process is very readily identified in this stain and it cannot be mistaken for anything else.

\section{SUMMARY}

Detailed reports of two new cases of progressive multifocal leucoencephalopathy are given. One had tiny, scattered lesions with abnormal oligodendroglia and was associated with an acute myelogenous leukaemia. The other had typical small and large lesions and was associated with chronic lymphatic leukaemia. The presence in the brain of leukaemic infiltration and plasma cells and morular bodies were interesting features of the second case. Reasons are given in support of the viral aetiology of this disease.

We are indebted to Dr. E. P. Richardson (1964) for reviewing our material, to Dr. Jon Kosek for performing some of the special stains on the morular cells, and to Mr. Wilmer Renner for the photography.

\section{ADDENDUM}

Since this paper was completed, a further case of progressive multifocal leucoencephalopathy has been studied by Silverman and Rubinstein at Stanford School of Medicine. The findings, which strongly endorse the viral origin of the disease, will be reported elsewhere.

\section{REFERENCES}

Åström, K. E., Mancall, E. L., and Richardson, E. P. Jr. (1958). Progressive multifocal leuko-encephalopathy: a hitherto unrecognized complication of chronic lymphatic leukaemia and Hodgkin's disease. Brain, 81, 93-111.

Bird, T., Ennis, J. E., Wort, A. J., and Gardner, P. S. (1963). Disseminated herpes simplex in newborn infants. J. clin. Path., 16, 423-431.

Cavanagh, J. B., Greenbaum, D., Marshall, A. H. E., and Rubinstein, L. J. (1959). Cerebral demyelination associated with disorders of the reticuloendothelial system. Lancet, 2, 524-529.

D'Agostino, A. N., Pease, G. L., and Kernohan, J. W. (1963). Cerebral demyelination associated with polycythemia vera. J. Neuropath. exp. Neurol., 22, 138-147.

Deep, W. D., Fraumeni, J. F., Tashima, C. K., and McDivitt, R. (1964). Leukoencephalopathy and dermatomyositis in Hodgkin's disease. Arch. intern. Med., 113, 635-640.

Headington, J. T., and Umiker, W. O. (1962). Progressive multifocal leukoencephalopathy. Neurology (Minneap.), 12, 434-439.

Hecker, R., and Reid, R. T. W. (1962). Cerebral demyelination in Whipple's disease. Med. J. Aust., 1, 211-212.

Janssen, P., Bogaert, L. van, and Haymaker, W. (1956). Pathology of the peripheral nervous system in African trypanosomiasis. J. Neuropath. exp. Neurol., 15, 269-287.

Mott, F. W. (1907). Histological observations on the changes in the nervous system in trypanosome infections, especially sleeping sickness and dourine, and their relation to syphilitic lesions of the nervous system. Arch. Neurol. (Lond.), 3, 581-646.

Richardson, E. P. Jr. (1961). Progressive multifocal leukoencephalopathy. New Engl.J. Med., 265, 815-823.

- (1964). Personal communication.

Rubinstein, L. J. (1964). Personal communication.

Spielmeyer, W. (1922). Histopathologie des Nervensystems. Springer, Berlin.

Waksman, B. H., and Adams, R. D. (1962a). A histologic study of the early lesion in experimental allergic encephalomyelitis in the guinea pig and rabbit. Amer. J. Path., 41, 135-162.

(1962b). Infectious leukoencephalitis. J. Neuropath. exp. Neurol., 21, $491-518$.

Weinstein, V. F., Woolf, A. L., and Meynell, M. J. (1963). Progressive multifocal leucoencephalopathy and primary hypersplenism. J. clin. Path., 16, 405-418.

Welsh, R. A. (1962). Light and electron microscopic correlation of the periodic acid-Schiff reaction in the human plasma cell. Amer. J. Path., 40, 285-296. 